Mappemonde

Revue trimestrielle sur l'image géographique et les formes du territoire

$128 \mid 2020$

Varia

\title{
CartOrient Cartes et textes - Asie occidentale - Caucase - Asie centrale
}

Ségolène Débarre

\section{OpenEdition}

Journals

Édition électronique

URL : http://journals.openedition.org/mappemonde/3997

DOI : 10.4000/mappemonde.3997

ISSN : 1769-7298

Éditeur

UMR ESPACE

Référence électronique

Ségolène Débarre, «CartOrient Cartes et textes - Asie occidentale - Caucase - Asie centrale ",

Mappemonde [En ligne], 128 | 2020, mis en ligne le 01 juillet 2020, consulté le 15 septembre 2020.

URL : http://journals.openedition.org/mappemonde/3997

Ce document a été généré automatiquement le 14 septembre 2020.

\section{(c) (i) (2)(2)}

La revue Mappemonde est mise à disposition selon les termes de la Licence Creative Commons Attribution - Pas d'Utilisation Commerciale - Partage dans les Mêmes Conditions 4.0 International. 


\title{
CartOrient Cartes et textes - Asie occidentale - Caucase - Asie centrale
}

\author{
Ségolène Débarre
}

1 Des cartes historiques conservées dans les archives nationales géorgiennes à la présence contemporaine des Ouïghours au Kazakhstan, de l'analyse des résultats d'élections présidentielles en Iran à la compréhension des fluctuations des taux de divorces au Caucase, les possibilités offertes par le site Cartorient (http:// cartorient.cnrs.fr/) sont riches et surprenantes. Héritier de l'expérience menée sur l'espace iranien par le géographe Bernard Hourcade (http://www.irancarto.cnrs.fr/), CartOrient couvre depuis 2018 un vaste espace centrasiatique, en s'appuyant sur une équipe pluridisciplinaire composée d'historiens, de géographes, d'un anthropologue, d'un linguiste et, bien sûr, de cartographes sous la direction de Julien Thorez, chargé de recherche au Centre de recherche Mondes iranien et indien (UMR 7528 CNRS). Tou'tes (une cartographe, Constance Lecomte, coordonne la publication) ont réuni leurs compétences pour proposer un site original à disposition d'un large public. Grâce au soutien du CNRS, de la Bibliothèque des langues et civilisations (BULAC) et de la Bibliothèque nationale de France (BNF), Cartorient est en effet accessible gratuitement, en français et en anglais.

2 Organisé en trois volets, le site propose un accès à des cartes historiques et des atlas numérisés conservés en France et à l'étranger; une interface de visualisation interactive accompagnée de textes inédits; des dossiers thématiques réalisés à partir de cartes et de textes consultables dans l'« atlas interactif ", au nombre de trois pour l'instant: l'Atlas des élections présidentielles en République islamique d'Iran (1980-2017) (Bernard Hourcade), l'Atlas du Caucase (Jean Radvanyi) et l'Atlas des minorités nationales au Kazakhstan (Julien Thorez). Il ne s'agit pas seulement d'utiliser des cartes pour illustrer un propos, mais d'argumenter cartographiquement afin de faire émerger des réalités qui, sans cette représentation, resteraient largement occultées. L'objectif est donc de valoriser une compréhension spatiale et multiscalaire des faits sociaux.

Prenons un exemple. 
Figure 1. Carte des divorces dans le Caucase en 2014

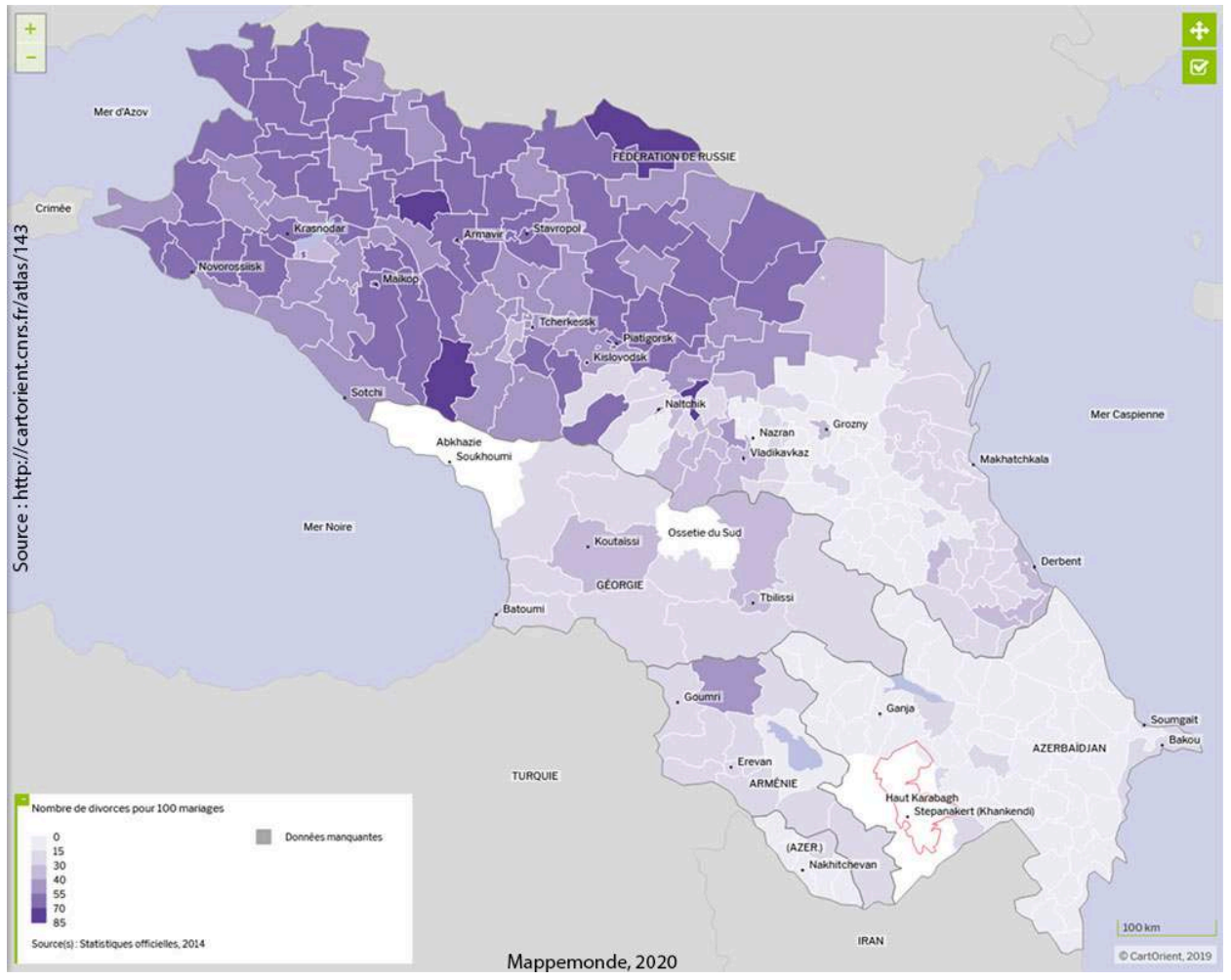

http://cartorient.cnrs.fr/atlas/143

4 La carte des divorces dans le Caucase en 2014 proposée par Jean Radvanyi ${ }^{1}$ permet d'observer que le nombre de divorces pour 100 mariages est plus élevé dans les régions où la population russe est la plus nombreuse : "pour les deux régions occidentales du Nord Caucase (Krasnodar et Stavropol), où les Russes sont majoritaires, [les indicateurs] suivent fidèlement la tendance moyenne de la Russie », pays où le taux de divorce est l'un des plus élevés au monde. Dans le reste du Caucase, l'indicateur est « pratiquement partout inférieur de moitié aux niveaux constatés dans le Caucase du Nord-Ouest», qu'il s'agisse de régions à majorité musulmane ou chrétienne, transcendant donc des différences culturelles et religieuses. La carte associée présentant la part des Russes dans la population du Caucase (régions et districts, 2010) permet de se convaincre de la corrélation. Si la démonstration est claire et efficace (le caractère synthétique des textes donne un indéniable attrait pédagogique à la plateforme), on peut seulement regretter que l'interactivité proposée soit limitée à la consultation des valeurs mesurées district par district. À terme, peut-être, le site permettra au lecteur de faire figurer et de croiser les variables de son choix. 
Figure 2. Carte présentant la part des Russes dans la population du Caucase

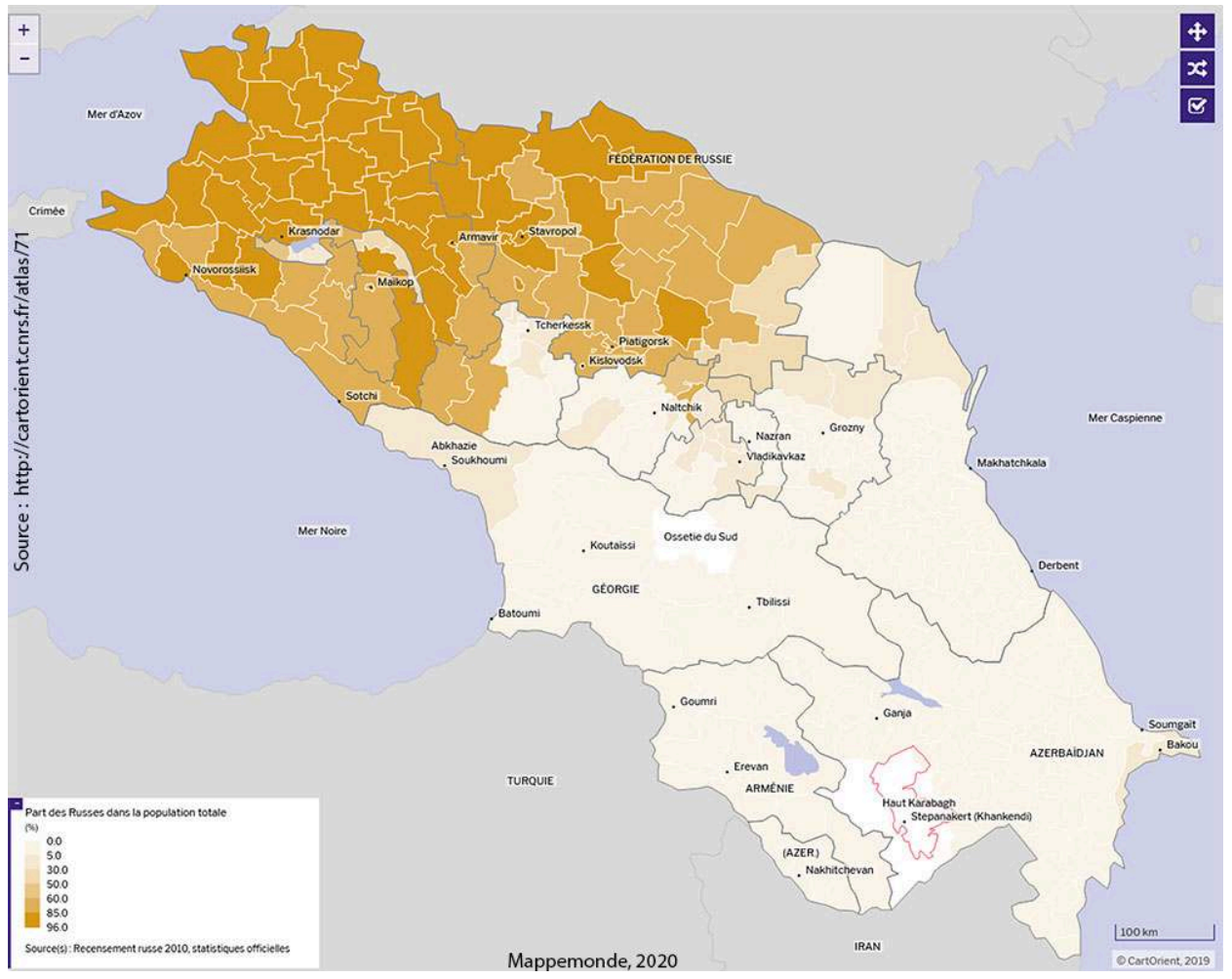

http://cartorient.cnrs.fr/atlas/71

5 Deux options s'offrent ensuite au lecteur : poursuivre ses recherches sur la région concernée en ouvrant l'atlas thématique du Caucase ou bien continuer par thème dans l'atlas interactif en s'intéressant, par exemple, à la population russe présente, cette fois, au Kazakhstan². Si la navigation est simple entre les cartes d'un même onglet (l'« atlas interactif », qui comprend actuellement 43 cartes, ou au sein des " atlas thématiques »), elle l'est moins d'un onglet à l'autre. Des liens pour faciliter la navigation manquent entre l' «atlas interactif » et la "bibliothèque d'atlas » comme le montre l'exemple suivant: un lecteur intéressé par l' "environnement" dans l'atlas interactif ne trouvera qu'une seule entrée (le très intéressant article relatif à «l'intensification du pastoralisme dans les steppes kazakhes des années 1930 aux années $1980 »^{3}$ ). Or, la bibliothèque d'atlas propose d'autres ressources sur le sujet, tel que l'Atlas des ressources naturelles de l'Asie centrale, l'Atlas écologique de l'Ouzbékistan ou encore l'Atlas environnemental de l'Ouzbékistan. Au lecteur, donc, de fouiller et de mener l'enquête dans chacun des onglets.

6 À l'intérieur de ces derniers, le curieux reste un peu sur sa faim lorsque son intérêt porte sur des rubriques non encore alimentées: par exemple «paysages", « migrations », « Afghanistan » ou encore «Irak-Kurdistan » dans l'atlas interactif, « Le poids des conflits persistants/Des inégalités sociales et économiques/Crise et développement : nouvelles tendances, nouveaux défis » dans l'atlas du Caucase). Mais le site étant récent, l'existence d'une telle indexation est une promesse d'avenir et une invitation aux contributions: Cartorient étant participatif, quiconque peut en effet soumettre une carte inédite pour l'alimenter et l'étoffer".

7 Pour remonter dans le temps, on se rend dans la «bibliothèque d'atlas " ${ }^{5}$ qui met à disposition du public des cartes et atlas numérisés et propose des liens vers des 
catalogues en France et à l'étranger : elle comporte actuellement 34 entrées (18 pour les éditions antérieures à 1950, 7 entre 1950 et 2000 et 14 après 2000). Chaque entrée peut renvoyer à un ensemble de cartes: par exemple la «collection de cartes de l'Afghanistan de la Bibliothèque nationale de France " offre à elle seule 55 cartes numérisées. Le champ des possibles est donc très vaste, reflétant en cela la dynamique actuelle de numérisation et de valorisation du patrimoine des bibliothèques françaises et étrangères ${ }^{6}$.

Aux cartes de la Bibliothèque nationale de France s'ajoutent des collections conservées en Russie, en Géorgie, en Grande-Bretagne ou aux États-Unis. On pourrait inclure, dans la liste existante, la récente " compilation de cartes scannées du XIX ${ }^{e}$ siècle » portant sur le "Moyen-Orient, l'Afrique du Nord et l'Asie centrale" mise en ligne par l'Université de Chicago'.

Parmi les cartes historiques, Cartorient permet ainsi de consulter la Carte de l'Asie centrale de Julius von Klaproth (1783-1835), éditée en 1828, qui a pour spécificité d'être la première occurrence cartographique de la région "centrasiatique ». Comme l'a bien montré Svetlana Gorshenina ${ }^{8}$, le toponyme d' "Asie centrale » a une histoire longue, depuis sa consécration par Alexandre de Humboldt, et une histoire mouvementée, du fait de concurrences et de chevauchements avec entre autres les termes de "Tartarie", " Turkestan », « Asie moyenne », « Touran » ou « Eurasie »; une histoire qui ne peut se comprendre qu'en prenant en considération les arrière-plans géopolitiques notamment russes - visant la région au XIX ${ }^{\mathrm{e}}$ et au $\mathrm{XX}^{\mathrm{e}}$ siècles.

Figure 3. Carte de l'Asie centrale

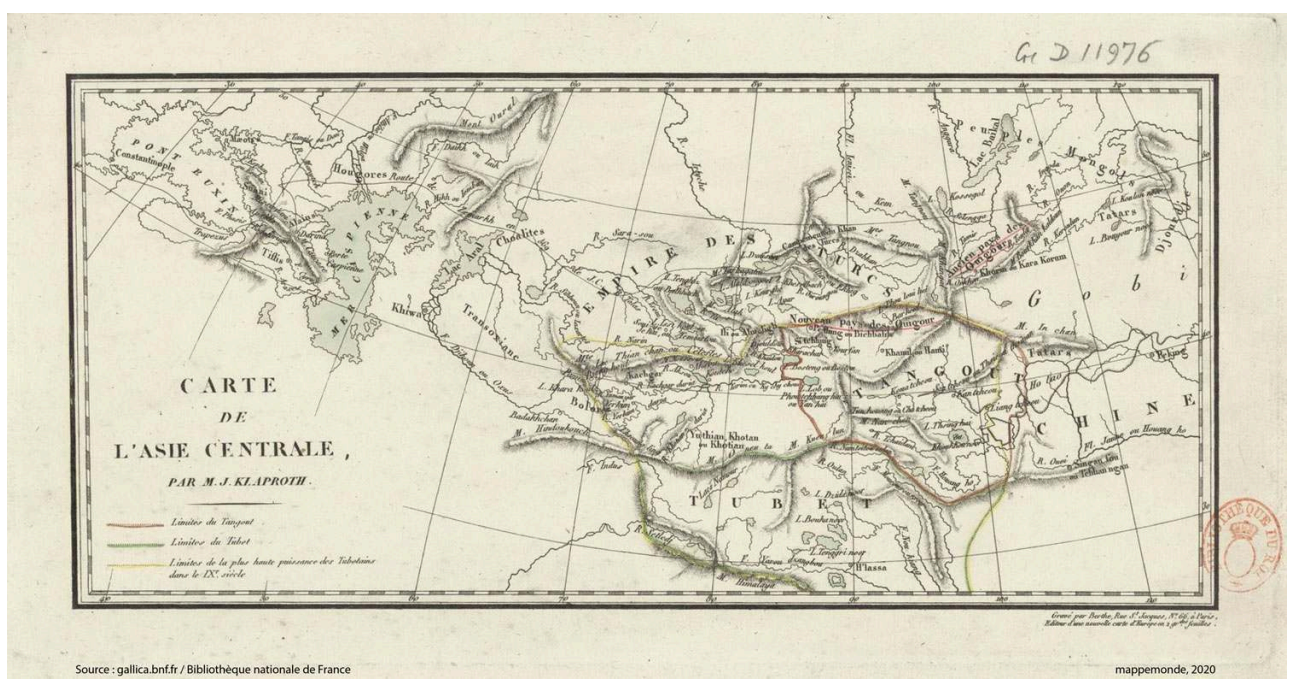

Julius von Klaproth, 1828 ; BnF

Pour saisir tout cela, il serait utile de fournir au lecteur, sinon une présentation détaillée et critique des documents historiques rassemblés, du moins quelques repères concernant les fonds cartographiques. Cela est particulièrement le cas pour les collections en russe ou en géorgien, moins connues d'un public hexagonal : que penser par exemple de cet " atlas de Russie asiatique » publié en 1914 et dont la description n'est accessible qu'en russe?

11 Si tout lecteur, étudiant, journaliste, entrepreneur ou simple curieux peut d'ores et déjà largement trouver de quoi alimenter ses connaissances et sa compréhension des 
sociétés centrasiatiques, quelques ajouts seraient les bienvenus (des liens entre les ressources, une présentation des cartes historiques, plus d'options d'interactivité), afin que tou'te's puissent s'emparer facilement du site et en retirer la substantifique moelle, au-delà des chercheurees spécialistes de la région.

12 Au-delà de ces quelques remarques, qui ne remettent nullement en cause la qualité et la portée de ce beau projet, il nous reste à espérer que cette plateforme en accès libre sera régulièrement enrichie et, pourquoi pas, élargie dans ses horizons géographiques à un plus proche ou plus lointain Orient.

\section{NOTES}

1. Jean Radvanyi, «Les divorces dans le Caucase en 2014 (régions et districts, 2014) », CartOrient. Mis en ligne le 17 Mai 2018. Consultation le 23 Février 2020. URL : http://cartorient.cnrs.fr/atlas/ 143

2. Julien Thorez, «Les Russes au Kazakhstan (districts, 2009)», CartOrient. Mis en ligne le 15 Janvier 2018. Consultation le 23 Février 2020. URL : http://cartorient.cnrs.fr/atlas/43

3. Isabelle Ohayon, «L'intensification du pastoralisme dans les steppes kazakhes (années 1930 années 1980) ", CartOrient. Mis en ligne le 07 Mars 2018. Consultation le 23 Février 2020. URL: http://cartorient.cnrs.fr/atlas/140

4. http://cartorient.cnrs.fr/accueil/contribuer

5. Sans doute serait-il plus simple pour le lecteur de distinguer clairement les feuillets cartographiques historiques d'atlas parfois très contemporains numérisés ou numériques.

6. Voir par exemple le projet « Bibliothèques d'Orient » : https://www.bnf.fr/fr/bibliothequesdorient

7. https://www.lib.uchicago.edu/e/collections/maps/middleeast19/

8. Svetlana Gorshenina. L'Invention de l'Asie centrale. Histoire du concept de la Tartarie à l'Eurasie. Genève, Droz, 2014, 704 p. ; Svetlana Gorshenina, «Comment penser l'Asie du Milieu et l'Asie du Centre ?», Espace populations sociétés, 2007/1 | 2007, p. 15-31. 